\title{
Study on Application of Distributed Fiber Optic Temperature Measurement System on Power Transmission Safety Testing
}

\author{
Minglin Liu ${ }^{1}$, Chenyu Zhao ${ }^{2}$, Nan Sun ${ }^{3}$ \\ ${ }^{1}$ State Grid Shandong Electric Power Company Maintenance Department, Shandong, 250001 \\ ${ }^{2}$ State Grid Shandong Electric Power Company Maintenance Department Shandong, 250001 \\ ${ }^{3}$ Bandweaver Technologies, Shanghai, 200000 \\ 43967125@163.com
}

Keywords: Distributed Fiber Optic; Temperature Measurement System; Power Transmission Safety Testing

\begin{abstract}
Cable is in the daily production, life everywhere. In power plants, factories, laboratories usually concentrated a large number of cables for easy wiring, maintenance, and beautiful. As people grow dependent on electricity, large concentrations of cable make the probability of fire accidents also increased. Current thermal effects will cause a current passes through the core of the heating cable and temperature continues to rise, rather than the normal duration of overheating or long-term work overload will make the cable insulation aging faster. When the condition reaches breakdown insulating protective layer, it will cause a fire, resulting in people's lives and property safety hazard. This paper studies the application of temperature on the light transmission of electrical safety and makes effort to improve the security level of automation power transmission, reduced power transmission risk.
\end{abstract}

\section{Introduction}

Compared with some developed countries, China has a high average failure rate of the grid system. One important reason is the lack of power-line monitoring system effectively. The temperature monitoring is one very important aspect, as can occur in an accident early warning through early temperature measurements and take prompt measures can effectively prevent such accidents. The traditional method is to point temperature sensing element such as a thermocouple attached to cable or other important parts of the power system temperature, or the use of fiber gratings and quasi-distributed measurements. These methods can only be a partial position in the power system temperature, but can not achieve temperature monitoring for the entire power system, and has some flaws in economic and practical terms. The distributed optical fiber temperature measurement system (Distributed Optical Fiber Temperature Sensor System) enables multi-point, line distributed measurement. It uses optical fiber to sense temperature information can be transmitted temperature information, high temperature, anti-electromagnetic radiation, high bandwidth and other characteristics, thus greatly enhancing the temperature and spatial resolution, effectively solve the high temperature for a long time the scene appears, Problems combustion, explosions and other emergency is not prepared. In the power system, this fiber temperature sensing technology in parts of high-voltage power cables, electrical equipment due to poor contact is easy to produce heat, cable sandwich, cable channels, a large generator stator, large transformers, boilers and other facilities designated sensing occasion It has broad application prospects.

\section{Fiber Optic Temperature Measurement Principle}

After the distributed optical fiber temperature measurement system based on the principle of backscatter can be divided after the Rayleigh scattering-based, based on the post-Raman scattering and the Brillouin scattering based distributed fiber optic temperature measurement systems. At present, more mature, and have products used in the project is based on the Raman scattering after Distributed Optical Fiber Temperature Measurement System. Its sensing principle is based mainly 
fiber optical time domain reflect (Optical Time Domain Renection, referred OTDR) principle and backward Raman scattering temperature effect, that is, the sensor technology for positioning OTDR, Raman scattering effect with temperature, so as to achieve a temperature and from the one to one relationship.

OTDR positioning principle is: When the laser pulse propagation in the fiber, due to the presence of microscopic in homogeneity of the refractive index of the optical fiber, will produce scattering. In the time domain, after the return to the scattering of incident light to the optical fiber entrance end of the time required for the t, laser pulses in optical fibers are moving when a laser pulse is injected into the optical fiber from one end of the fiber, the light pulses along the fiber direction before transmission. Since the elastic collision and inelastic collisions with the light pulse inside the fiber molecules, so the light pulse propagation in reflection in every point will be, among the reflected light reflected a small part, which coincides with the direction opposite to the direction of the incident light (also called backward). There is a certain correlation between the temperature and light intensity of the reflected light of the reflection point of this post. That is, after the intensity of the reflected light may reflect the temperature of the reflection point. Using this phenomenon, if we measure the intensity of light reflected back, we can calculate the temperature of the reflection point, which is the basic principle of the use of optical fiber for measuring temperature.

Specific expression of distributed optical fiber temperature measurement system analysis and mathematical models in physical terms that the laser pulse propagation in the fiber, the fiber molecules and photons can interact thermal vibration energy exchange. Specifically, if a portion of the light energy is converted into thermal vibration, it will emit a longer wavelength than the light source, known as La Ostocks (Raman Stokes) light; if a portion of the thermal vibration is converted to light, then it will issue a than the light wave length of light, called anti-Stokes Raman (Raman Anti-Stokes) light. They are distributed in the spectrum diagram roughly symmetrical, they are sensitive to temperature, but the anti-Stokes Raman scattering coefficient temperature sensitive Bithtocks Raman scattering is much greater. Thus, in general they are used as anti-Stokes Raman scattering signal path, as the main basis for calculating temperatures. The Stokes Raman scattering is generally used as the reference channel, to eliminate stress other factors.

\section{Distributed optical fiber temperature sensing system structure and process}

The hardware part of distributed optical fiber temperature measurement system consists of portions and the circuit portion of the light path: the path portion includes a pulsed laser light and its driver, directional couplers, optical filters and photodetectors; circuit part to the former multi-stage amplifiers, high-speed data acquisition card, the synchronization control circuit. Software part is mainly written by the computer system and display software finishes reading the data acquisition card status, Stokes data, Anti stokes a data and environmental temperature and other data; by calculation, calculate cable temperature data at each point in the temperature field and displays a complete temperature profile. In addition, pre-set by the database illustrates the value and alarm system linkage, issued a timely warning.

Consistent computer sends a signal to the synchronization control circuit, under the control of the synchronization pulse laser and simultaneously start the data acquisition card, so that the data collected in time (also can be understood as space) on: the sensing system is distributed fiber optic temperature measurement . Laser pulses through a directional coupler into the sensing fiber disaster, after the resulting scattered light back to the directional coupler acquisition by disaster film interference filters specific wavelengths of light into the rear light scattering Stokes Anti-Stokes light. By photo detector (generally used avalanche diode APD, mainly used for weak signal detection) to convert an optical signal into an electrical signal (for easy acquisition and processing), and then by broadband, forward low-noise amplifier circuit amplifies the signal to a suitable data acquisition card input signal amplitude, and finally stored and processed by the data acquisition card, complete a measured temperature curve. Due to affect the entire system losses and noise, the need for multiple measurements, the data accumulated, averaged to obtain the temperature field to better reflect the true temperature of the temperature curve. 
Real-time monitoring of the temperature profile data demodulation by acquiring the temperature acquiring unit, and displays the acquired measurement data in the form of temperature distribution curve. DTS temperature resolution of $0.1{ }^{\circ} \mathrm{C}$, the spatial resolution $\mathrm{lm}$, temperature accuracy disabilities $0.5{ }^{\circ} \mathrm{C}$. The system can be interconnected with other control devices via RS232 standard interface and relay output, so the temperature data and alarm signal upload. You can set multi-level fixed temperature alarm, such as early warning $30{ }^{\circ} \mathrm{C}, 40{ }^{\circ} \mathrm{C}$ pre-alarm, $50{ }^{\circ} \mathrm{C}$ take measures, and can be corrected for different site environments. Since the detection of the cable against electromagnetic interference, instant strong current, humidity and corrosion effects and multi-level set by the temperature difference between mild comprehensive comparison of two methods to determine the presence of fire, it does not produce false positives.

\section{Distributed Fiber Optic Temperature Measurement System in Power System}

High-voltage transmission cable lines can play a role in the power system, often because of long-running cable and insulation aging, due to which the adverse external environment and internal high load currents caused by localized high temperatures and even fire. Flexural properties using fiber glass fiber bend radius allowed in case, the temperature along the fiber optic cable lines were laid and close. In special circumstances and the important parts of the mark, then you can set the alarm threshold monitoring software. Cable joints often caused by a short circuit due to overload the joints, causing a fire or explosion. Since the high voltage cable is longer, and there are several joints, each joint is treated as key areas. By curvilinear relationship with the temperature of the load current to adjust the load current of the entire circuit, in order to support the safe operation of the power supply system. In addition, such as a cable trough, cable tunnels and other cable-intensive areas also need to focus on monitoring. The level of its internal ambient temperature to ensure the normal operation of the cable to have a great relationship and, if necessary, and ventilation and air conditioning systems with the use, in order to meet seasonal temperature at different situations, always ensure that the ambient temperature is within the allowable range.

$10 \mathrm{kv}, 35 \mathrm{kv}$ switchgear and moving contacts, switch cabinet connection (PT, CT, drainage lines, etc.), bus and a junction devices, etc., due to the long run, and the contact reliability deteriorates; because of the contact resistance larger at high heating power large currents, causing overheating. Since the optical fiber distributed optical fiber temperature measurement system using standard communication with multimode fiber, once fiber outer surface coated with different materials (such as acrylic acid salts, polymers, etc.), its temperature range can be extended to a $190{ }^{\circ} \mathrm{C}$ to $460{ }^{\circ} \mathrm{C}$, while anti-electromagnetic radiation characteristics of the fiber has, which makes it capable of operating in high voltage electrical installations such high temperatures, high pressure and intense radiation in the environment, and does not affect normal operation of these devices bring any negative impact. This is the traditional temperature measurement methods, such as thermocouples, infrared detectors can not match. As for the generator windings, the relatively large size of the transformer this important component can be an optical fiber is wound on the surface, in order to achieve the target temperature quickly find the point of failure in the temperature curve.

Currently, maintenance of electrical equipment is planned maintenance, engineering capacity, manpower, material and financial resources is high, the failure rate is also high cost. The distributed optical fiber temperature measurement system can provide a lot of scientific data that can be targeted to the maintenance department of electrical equipment condition-based maintenance, saving a lot of maintenance costs and management costs, reduce maintenance accident rate, improving the reliability of power supply. In addition, fiber optic distributed temperature measurement system can also monitor the number of power plant auxiliary equipment, such as silos, bridge, coal conveyor belt, boilers and the like. The safe operation of these devices is essential for the safe operation of power system conditions. 


\section{Conclusion}

Distributed optical fiber temperature measurement system has successfully solved the multi-line real-time monitoring of temperature problem, providing a powerful guarantee for the safe operation of the entire power system. Prospect for the future development and application of distributed optical fiber temperature measurement system is very broad, but its temperature resolution, spatial resolution and response times and other technical parameters are to be improved.

\section{References}

[1] M. Hobel, J. Richa, M. Wuthrich. High Resolution Distributed Temperature Sensing with the Multiphoto-Timing Technique. Applied Optics. 1995,34(16):2955-2967.

[2] K .Hotate, T.Hasegawa. Measurement of Brillouingain Spectrum Distributed Along Anoptical Fiber Using Acorrelation Based Technique Proposal Experiment Andsimulation. Photonics Technology Letters, 2000,83(3):405-412.

[3] Chruk Pall, G.Macphail. Application of Distributed Fiber Optic Temperature Sensing Onhydro's 525 Kv Submarine Cable System. High Voltage Science Technology, 2006, 59(12): 179-182.

[4] K. Hotate, M. Tanska. Distributed Fiber Sensing with $1 \mathrm{~cm}$ Spatial Resolution By Correlation Based Continuous-Wave Technique. Photon Technology Letter,2002,14(2):179-181. 